\title{
THE 2-RANK OF THE CLASS GROUP OF SOME REAL CYCLIC QUARTIC NUMBER FIELDS
}

\author{
ABDELMALEK AZIZI, MOHAMMED TAMIMI, AND ABDELKADER ZEKHNINI
}

\begin{abstract}
In this paper, we investigate the 2-rank of the class group of some real cyclic quartic number fields. Precisely, we consider the case where the quadratic subfield is $\mathbb{Q}(\sqrt{\ell})$ with $\ell \equiv 5(\bmod 8)$ is a prime.
\end{abstract}

\section{Introduction}

Let $K$ be a number field and $H$ its 2-class group, that is the 2-Sylow subgroup of the ideal class group of $K$. The rank $r_{2}(H)$ of $H$ is the number of cyclic 2groups appearing in the decomposition of $H$ whose orders are a 2 power, that is the dimension of the $\mathbb{F}_{2}$-vector space $H / H^{2}$, where $\mathbb{F}_{2}$ is the field of 2 elements.

Many mathematicians are interested in determining $r_{2}(H)$ and the power of 2 dividing the class number of $K$. Hasse [12, Bauer [5] and others gave methods to determine the exact power of 2 dividing the class number of a quadratic number field. These methods were developed by C. J. Parry and his co-authors to determine $r_{2}(H)$ and the power of 2 dividing the class number of some imaginary cyclic quartic number field $K$ having a quadratic subfield $k$ with odd class number (e.g., [6, 17, 19, 20, 21]). For this, they needed a suitable genus theory convenient to their situation. Hence they showed that the theory firstly developed by Hilbert ([14]), assuming an imaginary base field $k$, can be adapted to the situation where $K$ is a totally imaginary quartic cyclic extension of a totally real quadratic subfield $k$. This theory can be applied, with minor modifications, to any quartic number field $K$ having a quadratic subfield $k$ of odd class number.

The 2-rank of any biquadratic number field $K$ is determined (partially or totally) in many paper $([17,18,6,17,2,3])$ up to the case: $K$ is a real quartic cyclic extension of the rational number field $\mathbb{Q}$. Denote by $k=\mathbb{Q}(\sqrt{\ell})$ its unique quadratic subfield, we aim to investigate this case, whenever $k$ has odd class number and the norm of its fundamental unit equals -1 , i.e. $\ell=2$ or $\ell$ is a prime congruent to $1(\bmod 4)$. In this paper, we restrict ourselves to the case where $\ell$ is a prime congruent to 5 (mod 8) for two reasons: to avoid long paper and the technics used in this case are little bit different from those used in the other cases.

An outline of the paper is as follows. In $\S 2$ we summarize some preliminary results on quartic cyclic number fields and the ambiguous class number formula, which we will use later. The main theorems are presented and proved in $\S 3$, In $\S$ 4 we characterize all real cyclic quartic number fields $K=\mathbb{Q}\left(\sqrt{n \epsilon_{0} \sqrt{\ell}}\right)$, with $l$ is a prime congruent to $5(\bmod 8)$, having a 2-class group trivial, cyclic, of rank 2 or of rank 3.

2000 Mathematics Subject Classification. 11R16; 11R29; 11R11; 11R80.

Key words and phrases. Real cyclic quartic number field, 2-rank, 2-class group, quadratic fields. 


\section{Notations}

Throughout this paper, we adopt the following notations.

- $\mathbb{Q}$ : the rational field.

- $\ell$ : a prime integer congruent to 5 modulo 8 .

- $k=\mathbb{Q}(\sqrt{\ell})$ : a quadratic field.

- $\epsilon_{0}$ : the fundamental unit of $k$.

- $n$ : a square-free positive integer relatively prime to $\ell$.

- $\delta=1$ or 2 .

- $d=n \epsilon_{0} \sqrt{\ell}$.

- $\mathbb{K}=k(\sqrt{d})$ : a real quartic cyclic number field.

- $\mathcal{O}_{k}\left(\right.$ resp. $\left.\mathcal{O}_{\mathbb{K}}\right)$ : the ring of integers of $k$ (resp. $\mathbb{K}$ ).

- $H$ : the 2-class group of $\mathbb{K}$.

- 2: the prime ideal of $k$ above 2 .

- $r_{2}(H)$ : the rank of $H$.

- $\mathbb{K}^{*}, k^{*}$ : the nonzero elements of the fields $\mathbb{K}$ and $k$ respectively.

- $N_{\mathbb{K} / k}(\mathbb{K})$ : elements of $k$ which are norm from $\mathbb{K}$.

- $p, q, p_{i}, q_{j}$ : odd prime integers.

- $\left(\frac{x, y}{p}\right)_{k}$ : quadratic norm residue symbol over $k$.

- $\left[\frac{\alpha}{\beta}\right]$ : quadratic residue symbol for $k$.

- $\left(\frac{a}{b}\right)$ : quadratic residue (Legendre) symbol.

- $\left(\frac{a}{b}\right)_{4}$ : rational 4 -th power residue symbol.

\section{Preliminary results}

Let $K$ be a cyclic quartic extension of the rational number field $\mathbb{Q}$. By 13 , Theorem 1], it is known that $K$ can be expressed uniquely in the form $K=$ $\mathbb{Q}(\sqrt{a(\ell+b \sqrt{\ell})})$, where $a, b, c$ and $\ell$ are integers satisfying the conditions: $a$ is odd and square-free, $\ell=b^{2}+c^{2}$ is square-free, positive and relatively prime to $a$, with $b>0$ and $c>0$. Note that $K$ possesses a unique quadratic subfield $k=\mathbb{Q}(\sqrt{\ell})$. Assuming the class number of $k$ odd and $N_{k / \mathbb{Q}}\left(\epsilon_{0}\right)=-1$, then, by [22], this is equivalent to that $K / k$ has an integral basis, and by [15], this is equivalent to the existence of an integer $n$ such that $K=\mathbb{Q}\left(\sqrt{n \epsilon_{0} \sqrt{\ell}}\right)$ with $\epsilon_{0}$ is the fundamental unit of $k$, and

$$
n=\left\{\begin{aligned}
2 a & \text { if } \ell \equiv 1(\bmod 4) \text { and } b \equiv 1(\bmod 2) \\
a & \text { otherwise }
\end{aligned}\right.
$$

Recall that the extensions $K / \mathbb{Q}$ were investigated by Hasse in a paper ([11]) prior to that of Leopoldt ([16]) on the arithmetic interpretation of the class number of real abelian fields. They were also investigated by M. N. Gras [8, 9, 10, ...] and others. We have the following remark.

Remark 2.1. Keeping the notations above of a cyclic quartic field, $K$ is then real if and only if $a>0$ (equivalently $n>0$ ).

The field $K$ also satisfies the following lemma.

Lemma 2.1 ([23]). Let $a, b$ and $c$ be positive integers, $\ell=b^{2}+c^{2}$, with a and $c$ odd, then $\mathbb{Q}(\sqrt{2 a(\ell+b \sqrt{\ell}}))=\mathbb{Q}(\sqrt{a(\ell+c \sqrt{\ell}})$. 
We will need the following theorem about the conductor $f_{K}$ of $K$.

Theorem 2.1 ([13]). The conductor $f_{K}$ of the (real or imaginary) cyclic quartic field $K=\mathbb{Q}(\sqrt{a(\ell+b \sqrt{\ell}})$, where $a$ is an odd square-free integer, $\ell=b^{2}+c^{2}$ is a square-free positive integer relatively prime to $a$, with $b>0$ and $c>0$, is given by $f_{\mathbb{K}}=2^{e}|a| \ell$, where $e$ is defined by:

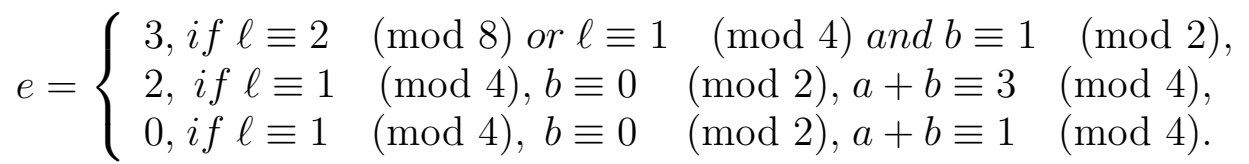

We end this section by recalling the number of ambiguous ideal classes of a quadratic extension $K / k$.

Theorem 2.2 ([1, 19]). Let $K / k$ be a cyclic extension of prime degree $p$. Denote by $A_{K / k}$ the number of ambiguous ideal classes of the number field $K$ with respect to $k$, then:

with:

$$
A_{K / k}=h(k) 2^{\mu+r^{*}-(r+c+1)}
$$

$r$ the number of the fundamental units of $k$.

$\mu$ the number of prime ideals of $k$ (finite or infinite) which ramify in $K$.

$r^{*}$ is defined by $2^{r^{*}}=\left[N_{K / k}\left(K^{*}\right) \cap E_{k}: E_{k}^{2}\right]$ with $E_{k}$ is the group of units of $k$.

$c=1$ if $k$ contains a primitive $p$-th root of unity and $c=0$ otherwise.

Furthermore, if $p=2$ and the class number of $k$ is odd, then the 2-rank of the class group of $K$ is equal to

$$
\mu+r^{*}-(r+c+1) .
$$

The remark below explain how to get $r^{*}$.

Remark 2.2. Since the unit group of $k$ is generated by -1 and $\epsilon_{0}$, so

- $r^{*}=0$, if $-1, \epsilon_{0}$ and $-\epsilon_{0}$ are not in $N_{\mathbb{K} / k}\left(\mathbb{K}^{*}\right)$.

- $r^{*}=1$, if $\left(-1\right.$ is in $N_{\mathbb{K} / k}\left(\mathbb{K}^{*}\right)$ and $\epsilon_{0}$ is not $)$ or $\left(-1\right.$ is not in $N_{\mathbb{K} / k}\left(\mathbb{K}^{*}\right)$ and $\epsilon_{0}$ or $-\epsilon_{0}$ is $)$.

- $r^{*}=2$, if -1 and $\epsilon_{0}$ are in $N_{\mathbb{K} / k}\left(\mathbb{K}^{*}\right)$.

\section{THE RANK OF $H$}

Let $\ell$ be a prime integer congruent to $5(\bmod 8)$ and $n$ a square-free positive integer relatively prime to $\ell$. Let $\mathbb{K}=k\left(\sqrt{n \epsilon_{0} \sqrt{\ell}}\right)$ and $k=\mathbb{Q}(\sqrt{\ell})$, where $\epsilon_{0}$ is the fundamental unit of $k$.

On one hand, as $\ell \equiv 1(\bmod 4)$, so it is well known (e.g., [23]) that $\epsilon_{0}=\frac{u+v \sqrt{\ell}}{2}$ and $N\left(\epsilon_{0}\right)=\frac{u+v \sqrt{\ell}}{2} \frac{u-v \sqrt{\ell}}{2}=\frac{u^{2}-v^{2} \ell}{4}=-1$. Since $u+v \sqrt{\ell}>0$, then $u-v \sqrt{\ell}<0$. On the other hand, the polynomial $f(x)=x^{4}-m v \ell x^{2}+m^{2} \ell$ is irreducible. Indeed, as $\ell$ divides both of $m v \ell$ and $m^{2} \ell$, and $\ell^{2}$ does not divide $m^{2} \ell$ since $m$ and $\ell$ are relatively prime, so the Eisentein characterization implies our claim. Thus $f$ is the characteristic polynomial of $\mathbb{K}$. By the Kaplansky's theorem [4, page 68] and since $m^{2} \ell\left[(m v \ell)^{2}-4 m^{2} \ell\right]=m^{4} \ell^{2}\left(v^{2} \ell-4\right)=m^{4} \ell^{2} u^{2} \in \mathbb{Q}^{2}$, the polynomial $f$ defines a cyclic extension over $\mathbb{Q}$ of degree 4 , i.e., $\mathbb{K}$ is a real cyclic quartic number field and $k$ is its unique quadratic subfield.

To determine the exact value of $r^{*}$, we will use the norm residue symbol applied to primes of $k$ ramifying in $\mathbb{K}$. Note that the infinite prime ideals of $k$ don't ramify 
in the extension $\mathbb{K}$. Indeed, the discriminant of $g(X)=f\left(x^{2}\right)=X^{2}-m v \ell X+m^{2} \ell$ is

$$
\Delta=(m v \ell)^{2}-4 m^{2} \ell=m^{2} \ell\left(v^{2} \ell-4\right)=m^{2} \ell u^{2} \geq 0,
$$

hence the roots of $g$ are

$$
\begin{gathered}
X_{1}=x_{1}^{2}=\frac{m v \ell-m u \sqrt{\ell}}{2}=-m \sqrt{\ell}\left(\frac{u-v \sqrt{\ell}}{2}\right) \geq 0 \text { and } \\
X_{2}=x_{2}^{2}=\frac{m v \ell+m u \sqrt{\ell}}{2}=m \sqrt{\ell}\left(\frac{u+v \sqrt{\ell}}{2}\right)=m \epsilon_{0} \sqrt{\ell} \geq 0 .
\end{gathered}
$$

From which we deduce the result claimed.

Note that, as $n$ is relatively prime to $\ell$, so the prime integers dividing $n$ don't ramify in $k$. Likewise 2 stay inert in $k$ since $\ell \equiv 5(\bmod 8)$.

To compute $r_{2}(H)$, the rank of the 2-class group $H$ of $\mathbb{K}$, we will distinguish many cases. For this, let $p_{1}, p_{2}, \cdots, p_{t}, q_{1}, \cdots, q_{s}$ be positive prime integers. Put $\delta=1$ or 2 .

3.1. First case: $n=\delta \prod_{i=1}^{t} p_{i}$ and, for all $i, p_{i} \equiv 1(\bmod 4)$.

Theorem 3.1. Let $\mathbb{K}=\mathbb{Q}\left(\sqrt{n \epsilon_{0} \sqrt{\ell}}\right)$ be a real cyclic quartic number field, where $\ell \equiv 5(\bmod 8)$ is a positive prime integer, $n$ a square-free positive integer relatively prime to $\ell$ and $\epsilon_{0}$ the fundamental unit of the quadratic subfield $k=\mathbb{Q}(\sqrt{\ell})$. Let $n=\delta \prod_{i=1}^{i=t} p_{i}$ with $p_{i} \equiv 1(\bmod 4)$ for all $i \in\{1, \ldots, t\}$ and $t$ is a positive integer.

1. If, for all $i,\left(\frac{p_{i}}{\ell}\right)=-1$, then $r_{2}(H)=t$.

2. If, for all $i,\left(\frac{p_{i}}{\ell}\right)=1$, then $r_{2}(H)=2 t$.

Moreover, if $n=\delta \prod_{i=1}^{i=t_{1}} p_{i} \prod_{j=1}^{i=t_{2}} q_{j}$ with $\left(\frac{p_{i}}{\ell}\right)=-\left(\frac{q_{j}}{\ell}\right)=-1$ and $p_{i} \equiv q_{j} \equiv 1$ $(\bmod 4)$ for all $i \in\left\{1, \ldots, t_{1}\right\}$ and $j \in\left\{1, \ldots, t_{2}\right\}$, then $r_{2}(H)=t_{1}+2 t_{2}$.

Proof. To prove the theorem assertions, we have to compute the integer $r^{*}\left(2^{r^{*}}=\right.$ $\left.\left[N_{K / k}\left(K^{*}\right) \cap E_{k}: E_{k}^{2}\right]\right)$ by applying Remark [2.2, and then we call Theorem 2.2. To deduce primes of $k$ ramifying in $\mathbb{K}$, we use Theorem 2.1 .

1. If $\left(\frac{p_{i}}{\ell}\right)=-1$ for all $i=1, \ldots, t$, then the prime ideals of $k$ which ramify in $\mathbb{K}$ are $(\sqrt{\ell}), 2$ and the prime ideals $\mathfrak{p}_{i}, i=1, \ldots, t$, where 2 (resp. $\left.\mathfrak{p}_{i}\right)$ is the prime ideal of $k$ above 2 (resp. $p_{i}$ ), this implies that the number of primes of $k$ ramifying in $\mathbb{K}$ is $\mu=t+2$. Hence

$$
\begin{aligned}
& \left(\frac{-1, d}{\mathfrak{p}_{i}}\right)=\left[\frac{-1}{\mathfrak{p}_{i}}\right]=\left(\frac{-1}{p_{i}}\right)=1 \text { for all } i=1, \ldots, t . \\
& \left(\frac{-1, d}{(\sqrt{\ell})}\right)=\left[\frac{-1}{(\sqrt{\ell})}\right]=\left(\frac{-1}{\ell}\right)=1 . \\
& \left(\frac{\epsilon, d}{\mathfrak{p}_{i}}\right)=\left[\frac{\epsilon}{\mathfrak{p}_{i}}\right]=\left(\frac{-1}{p_{i}}\right)=1 \text { for all } i=1, \ldots, t . \\
& \left(\frac{\epsilon, d}{(\sqrt{\ell})}\right)=\left[\frac{\frac{u}{2}}{(\sqrt{\ell})}\right]=\left[\frac{2 u}{(\sqrt{\ell})}\right]=\left(\frac{2 u}{\ell}\right)=\left(\frac{2}{\ell}\right)\left(\frac{u}{\ell}\right)=-\left(\frac{\ell}{u}\right)=-1, \text { indeed as } \\
& \epsilon=\frac{u+v \sqrt{\ell}}{2}, \text { so }-4=u^{2}-v^{2} \ell .
\end{aligned}
$$

We summarize these results in the following table.

\begin{tabular}{|c|c|c|c|}
\hline Unit \Character & $\sqrt{\ell}$ & $\mathfrak{p}_{i}$ & $\mathbf{2}$ \\
\hline-1 & + & + & + \\
\hline$\epsilon$ & - & + & - \\
\hline$-\epsilon$ & - & + & - \\
\hline
\end{tabular}

So $r^{*}=1$, from which we infer that:

$$
r_{2}(H)=\mu+r^{*}-3=t+2+1-3=t .
$$


2. If $\left(\frac{p_{i}}{\ell}\right)=1$ for all $i=1, \ldots, t$, then the prime ideals of $k$ which ramify in $\mathbb{K}$ are $(\sqrt{\ell}), 2$ and the prime ideals $\wp_{i}$ and $\bar{\wp}_{i}$ with $p_{i} \mathcal{O}_{k}=\wp_{i} \bar{\wp}_{i}, i=1, \ldots, t$, in this case $\mu=2 t+2$. Hence

$$
\begin{aligned}
\left(\frac{-1, d}{\wp_{i}}\right)=\left(\frac{-1, d}{\wp_{i}}\right) & =\left[\frac{-1}{\wp_{i}}\right]=\left(\frac{-1}{p_{i}}\right)=1, \text { for all } i=1, \ldots, t . \\
\left(\frac{\epsilon, d}{\wp_{i}}\right) & =\left[\frac{\epsilon}{\wp_{i}}\right], \text { for all } i=1, \ldots, t .
\end{aligned}
$$

To compute the last unity, put $p_{i}^{h_{0}}=\wp_{i} \bar{\wp}_{i}$ and $\wp_{i}=a_{i}+b_{i} \sqrt{\ell}$ and $\bar{\wp}_{i}=a_{i}-b_{i} \sqrt{\ell}$, for all $i$ (note that $\mathcal{O}_{k}$ is a principal ring). According to [6] we have $\left[\frac{\epsilon \sqrt{\ell}}{\wp_{i}}\right]=\left(\frac{p_{i}}{\ell}\right)_{4}$. Thus

$$
\left[\frac{\epsilon}{\wp_{i}}\right]=\left(\frac{p_{i}}{\ell}\right)_{4}\left[\frac{\sqrt{\ell}}{\wp_{i}}\right]
$$

On the other hand,

$$
\left[\frac{\sqrt{\ell}}{\wp_{i}}\right]=\left[\frac{b_{i}^{2} \sqrt{\ell}}{\wp_{i}}\right]=\left[\frac{b_{i}\left(-a_{i}+a_{i}+b_{i} \sqrt{\ell}\right)}{\wp_{i}}\right]=\left[\frac{-a_{i} b_{i}}{\wp_{i}}\right]=\left(\frac{a_{i}}{p_{i}}\right)\left(\frac{b_{i}}{p_{i}}\right) .
$$

As $p_{i}^{h_{0}}=a_{i}^{2}-b_{i}^{2} \ell$, so $b_{i}^{2} \ell \equiv a_{i}^{2}\left(\bmod p_{i}\right)$. Note that $\ell$ and $p_{i}$ are relatively prime, then $b_{i}^{2} \ell^{2} \equiv \ell a_{i}^{2}\left(\bmod p_{i}\right)$, this implies that $\left(\frac{b_{i}}{p_{i}}\right)=\left(\frac{b_{i} \ell}{p_{i}}\right)=\left(\frac{b_{i}^{2} \ell^{2}}{p_{i}}\right)_{4}=\left(\frac{\ell a_{i}^{2}}{p_{i}}\right)_{4}=$ $\left(\frac{\ell}{p_{i}}\right)_{4}\left(\frac{a_{i}}{p_{i}}\right)$. Finally,

$$
\left[\frac{\epsilon}{\wp_{i}}\right]=\left(\frac{p_{i}}{\ell}\right)_{4}\left(\frac{a_{i}}{p_{i}}\right)\left(\frac{b_{i}}{p_{i}}\right)=\left(\frac{p_{i}}{\ell}\right)_{4}\left(\frac{a_{i}}{p_{i}}\right)\left(\frac{\ell}{p_{i}}\right)_{4}\left(\frac{a_{i}}{p_{i}}\right)=\left(\frac{p_{i}}{\ell}\right)_{4}\left(\frac{\ell}{p_{i}}\right)_{4} .
$$

Proceeding similarly, we get $\left[\frac{\epsilon}{\bar{\wp}_{i}}\right]=\left(\frac{p_{i}}{\ell}\right)_{4}\left(\frac{\ell}{p_{i}}\right)_{4}$. The two values $\left(\frac{-1, d}{(\sqrt{\ell})}\right)$ and $\left(\frac{\epsilon, d}{(\sqrt{\ell})}\right)$ are computed as above. These results are summarized in the following table:

\begin{tabular}{|c|c|c|c|c|}
\hline Unit \Character & $\sqrt{\ell}$ & $\wp_{i}$ & $\bar{\wp}_{i}$ & 2 \\
\hline-1 & + & + & + & + \\
\hline$\epsilon$ & - & $\left(\frac{p_{i}}{\ell}\right)_{4}\left(\frac{\ell}{p_{i}}\right)_{4}$ & $\left(\frac{p_{i}}{\ell}\right)_{4}\left(\frac{\ell}{p_{i}}\right)_{4}$ & - \\
\hline$-\epsilon$ & - & $\left(\frac{p_{i}}{\ell}\right)_{4}\left(\frac{\ell}{p_{i}}\right)_{4}$ & $\left(\frac{p_{i}}{\ell}\right)_{4}\left(\frac{\ell}{p_{i}}\right)_{4}$ & - \\
\hline
\end{tabular}

So $r^{*}=1$, from which we infer that:

$$
r_{2}(H)=\mu+r^{*}-3=2 t+2+1-3=2 t .
$$

If $n=\delta \prod_{i=1}^{i=t_{1}} p_{i} \prod_{j=1}^{i=t_{2}} q_{j}$ with $\left(\frac{p_{i}}{\ell}\right)=-\left(\frac{q_{j}}{\ell}\right)=-1$ for all $i \in\left\{1, \ldots, t_{1}\right\}$ and $j \in$ $\left\{1, \ldots, t_{2}\right\}$, then according to the two cases above, $r^{*}=1$ and $r_{2}(H)=t_{1}+2 t_{2}$.

\subsection{Second case: $n=1$ or 2 .}

Theorem 3.2. Let $\mathbb{K}=\mathbb{Q}\left(\sqrt{n \epsilon_{0} \sqrt{\ell}}\right)$ be a real cyclic quartic number field, where $\ell \equiv 5(\bmod 8)$ is a positive prime integer, $n$ a square-free positive integer relatively prime to $\ell$ and $\epsilon_{0}$ the fundamental unit of the quadratic subfield $k=\mathbb{Q}(\sqrt{\ell})$. If $n=1$ or 2 , then $r_{2}(H)=0$. 
Proof. As in the first case, we compute $r^{*}$ by applying Remark 2.2 , and then we call Theorem 2.2. For the two cases $n=1$ or 2, and by Theorem 2.1, the prime ideals of $k$ that ramify in $\mathbb{K}$ are $(\sqrt{\ell})$ and 2 , where 2 is the prime ideal of $k$ above 2, i.e. $\mu=2$. Proceeding as in the first case, we get the following table:

\begin{tabular}{|c|c|c|}
\hline Unit \Character & $\sqrt{\ell}$ & 2 \\
\hline-1 & + & + \\
\hline$\epsilon$ & - & - \\
\hline$-\epsilon$ & - & - \\
\hline
\end{tabular}

Hence $r^{*}=1$, which implies that $r_{2}(H)=\mu+r^{*}-3=2+1-3=0$.

3.3. Third case: $n=\prod_{i=1}^{i=t} p_{i}$ with $t$ is odd and, for all $i, p_{i} \equiv 3(\bmod 4)$.

Theorem 3.3. Let $\mathbb{K}=\mathbb{Q}\left(\sqrt{n \epsilon_{0} \sqrt{\ell}}\right)$ be a real cyclic quartic number field, where $\ell \equiv 5(\bmod 8)$ is a positive prime integer, $n$ a square-free positive integer relatively prime to $\ell$ and $\epsilon_{0}$ the fundamental unit of the quadratic subfield $k=\mathbb{Q}(\sqrt{\ell})$. Assume $n=\prod_{i=1}^{i=t} p_{i}, p_{i} \equiv 3(\bmod 4)$ for all $i=1, \ldots, t$ and $t$ is a positive odd integer.

1. If, for all $i,\left(\frac{p_{i}}{\ell}\right)=-1$, then $r_{2}(H)=t-1$.

2. If, for all $i,\left(\frac{p_{i}}{\ell}\right)=1$, then $r_{2}(H)=2 t-2$.

Moreover, if $n=\prod_{i=1}^{t_{1}} p_{i} \prod_{j=1}^{t_{2}} q_{j}$, where $p_{i} \equiv q_{j} \equiv 3(\bmod 4)$ and $\left(\frac{p_{i}}{\ell}\right)=-\left(\frac{q_{j}}{\ell}\right)=$ -1 , for all $i \in\left\{1, \ldots, t_{1}\right\}$, and for all $j \in\left\{1, \ldots, t_{2}\right\}$ with $t_{1}+t_{2}$ is odd, then $r_{2}(H)=t_{1}+2 t_{2}-2$.

Proof. As in the two cases above, we shall compute $r^{*}$ by applying Remark 2.2, and then we call Theorem 2.2. As above Theorem 2.1 gives us primes of $k$ that ramify in $\mathbb{K}$.

1. If $\left(\frac{p_{i}}{\ell}\right)=-1$ for all $i \in\{1, \ldots, t\}$, then the prime ideals of $k$ which ramify in $\mathbb{K}$ are $\mathfrak{p}_{i}$ and $(\sqrt{\ell})$, where $\mathfrak{p}_{i}$ is the prime ideal of $k$ above $p_{i}$. Hence, for all $i \in\{1, \ldots, t\}$, we have:

$$
\left(\frac{-1, d}{\mathfrak{p}_{i}}\right)=\left[\frac{-1}{\mathfrak{p}_{i}}\right]=\left(\frac{1}{p_{i}}\right)=1 \text { and }\left(\frac{\epsilon, d}{\mathfrak{p}_{i}}\right)=\left[\frac{\epsilon}{\mathfrak{p}_{i}}\right]=\left(\frac{-1}{p_{i}}\right)=-1 \text {. }
$$

The two values $\left(\frac{-1, d}{(\sqrt{\ell})}\right)$ and $\left(\frac{\epsilon, d}{(\sqrt{\ell})}\right)$ are computed as in the first case. We summarize these results in the following table:

\begin{tabular}{|c|c|c|}
\hline Unit \Character & $(\sqrt{\ell})$ & $\mathfrak{p}_{i}$ \\
\hline-1 & + & + \\
\hline$\epsilon$ & - & - \\
\hline$-\epsilon$ & - & - \\
\hline
\end{tabular}

Hence $r^{*}=1$, which implies that $r_{2}(H)=\mu+r^{*}-3=t+1+1-3=t-1$.

2. If $\left(\frac{p_{i}}{\ell}\right)=1$ for all $i \in\{1, \ldots, t\}$, then the prime ideals of $k$ which ramify in $\mathbb{K}$ are $(\sqrt{\ell}), \wp_{i}$ and $\bar{\wp}_{i}$, where $p_{i} \mathcal{O}_{k}=\wp_{i} \bar{\wp}_{i}, i=1, \ldots, t$. Hence, for all $i \in\{1, \ldots, t\}$, we have:

$$
\left(\frac{-1, d}{\wp_{i}}\right)=\left(\frac{-1, d}{\bar{\wp}_{i}}\right)=\left[\frac{-1}{\wp_{i}}\right]=\left(\frac{-1}{p_{i}}\right)=-1 .
$$

Proceeding as in the proof of Theorem 3.1, we get

$$
\left(\frac{\epsilon, d}{\wp_{i}}\right)=\left[\frac{\epsilon}{\wp_{i}}\right]=\left(\frac{p_{i}}{\ell}\right)_{4}\left(\frac{\ell}{p_{i}}\right)_{4} .
$$


According to [6], we have

On the other hand,

$$
\left[\frac{\epsilon \sqrt{\ell}}{\bar{\wp}_{i}}\right]=-\left(\frac{p}{\ell}\right)_{4} \text { then }\left[\frac{\epsilon}{\bar{\wp}_{i}}\right]=-\left(\frac{p}{\ell}\right)_{4}\left[\frac{\sqrt{\ell}}{\bar{\wp}_{i}}\right] .
$$

$$
\left[\frac{\sqrt{\ell}}{\bar{\wp}_{i}}\right]=-\left[\frac{-b_{i}^{2} \sqrt{\ell}}{\bar{\wp}_{i}}\right]=-\left[\frac{b_{i}\left(-a_{i}+a_{i}-b_{i} \sqrt{\ell}\right)}{\bar{\wp}_{i}}\right]=-\left[\frac{-a_{i} b_{i}}{\bar{\wp}_{i}}\right]=\left(\frac{a_{i}}{p_{i}}\right)\left(\frac{b_{i}}{p_{i}}\right),
$$

which implies (as in the proof of Theorem 3.1) that

$$
\left[\frac{\epsilon}{\bar{\wp}_{i}}\right]=-\left(\frac{p_{i}}{\ell}\right)_{4}\left(\frac{\ell}{p_{i}}\right)_{4}\left(\frac{a}{p_{i}}\right)^{2}=-\left(\frac{p_{i}}{\ell}\right)_{4}\left(\frac{\ell}{p_{i}}\right)_{4} .
$$

The two values $\left(\frac{-1, d}{(\sqrt{\ell})}\right)$ and $\left(\frac{\epsilon, d}{(\sqrt{\ell})}\right)$ are computed as above. We summarize these results in the following table:

\begin{tabular}{|c|c|c|c|}
\hline Unit/Character & $\sqrt{\ell}$ & $\wp_{i}$ & $\bar{\wp}_{i}$ \\
\hline-1 & + & - & - \\
\hline$\epsilon$ & - & $\left(\frac{p_{i}}{\ell}\right)_{4}\left(\frac{\ell}{p_{i}}\right)_{4}$ & $-\left(\frac{p_{i}}{\ell}\right)_{4}\left(\frac{\ell}{p_{i}}\right)_{4}$ \\
\hline$-\epsilon$ & - & $-\left(\frac{p_{i}}{\ell}\right)_{4}\left(\frac{\ell}{p_{i}}\right)_{4}$ & $\left(\frac{p_{i}}{\ell}\right)_{4}\left(\frac{\ell}{p_{i}}\right)_{4}$ \\
\hline
\end{tabular}

Hence $r^{*}=0$, which implies that:

$$
r_{2}(H)=\mu+r^{*}-3=2 t+1+0-3=2 t-2 .
$$

Finally, if $n=\prod_{i=1}^{t_{1}} p_{i} \prod_{j=1}^{t_{2}} q_{j}$, with $p_{i} \equiv q_{j} \equiv 3(\bmod 4)$ and $\left(\frac{p_{i}}{\ell}\right)=-\left(\frac{q_{j}}{\ell}\right)=-1$ for all $i \in\left\{1, \ldots, t_{1}\right\}$ and $j \in\left\{1, \ldots, t_{2}\right\}$ with $t_{1}+t_{2}$ is odd, then according to the two cases above, there are $t_{1}+2 t_{2}+1$ prime ideals of $k$ which ramify in $\mathbb{K}$ and $r^{*}=0$. Thus $r_{2}(H)=t_{1}+2 t_{2}+1+0-3=t_{1}+2 t_{2}-2$.

3.4. Fourth case: $n=\delta \prod_{i=1}^{i=t} p_{i}$ with $t$ is even or $n=2 \prod_{i=1}^{i=t} p_{i}$ with $t$ is odd and, for all $i, p_{i} \equiv 3(\bmod 4)$.

Theorem 3.4. Let $\mathbb{K}=\mathbb{Q}\left(\sqrt{n \epsilon_{0} \sqrt{\ell}}\right)$ be a real cyclic quartic number field, where $\ell \equiv 5(\bmod 8)$ is a positive prime integer, $n$ a square-free positive integer relatively prime to $\ell$ and $\epsilon_{0}$ the fundamental unit of the quadratic subfield $k=\mathbb{Q}(\sqrt{\ell})$. Assume $n=\delta \prod_{i=1}^{i=t} p_{i}$ and $t$ is even or $n=2 \prod_{i=1}^{i=t} p_{i}$ and $t$ is odd with $p_{i} \equiv 3(\bmod 4)$, $i=1, \ldots, t$.

1. If, for all $i,\left(\frac{p_{i}}{\ell}\right)=-1$, then $r_{2}(H)=t$.

2. If, for all $i,\left(\frac{p_{i}}{\ell}\right)=1$, then $r_{2}(H)=2 t-1$.

Moreover, if $n=\delta \prod_{i=1}^{t_{1}} p_{i} \prod_{j=1}^{t_{2}} q_{j}$, with $t_{1}+t_{2}$ is even, or $n=2 \prod_{i=1}^{t_{1}} p_{i} \prod_{j=1}^{t_{2}} q_{j}$ with $t_{1}+t_{2}$ is odd, where $p_{i} \equiv q_{j} \equiv 3(\bmod 4)$ and $\left(\frac{p_{i}}{\ell}\right)=-\left(\frac{q_{j}}{\ell}\right)=-1$, for all $i \in\left\{1, \ldots, t_{1}\right\}$ and $j \in\left\{1, \ldots, t_{2}\right\}$, then $r_{2}(H)=t_{1}+2 t_{2}-1$.

Proof. We proceed as above, we first compute $r^{*}$ by applying Remark 2.2, and then we apply Theorem 2.2. It is a routine, as above, to use Theorem 2.1 to get primes of $k$ that ramify in $\mathbb{K}$.

1. If $\left(\frac{p_{i}}{\ell}\right)=-1$ for all $i \in\{1, \ldots, t\}$, then the prime ideals of $k$ which ramify in $\mathbb{K}$ are $2, \mathfrak{p}_{i}$ and $(\sqrt{\ell})$, where $\mathfrak{p}_{i}$ (resp. 2) is the prime ideal of $k$ above $p_{i}$ (resp. 2). Proceeding as in the cases above, we get the following table. 


\begin{tabular}{|c|c|c|c|}
\hline Unit/Character & $\sqrt{\ell}$ & 2 & $\mathfrak{p}_{\mathfrak{i}}$ \\
\hline-1 & + & + & + \\
\hline$\epsilon$ & - & $(-1)^{t+1}$ & - \\
\hline$-\epsilon$ & - & $(-1)^{t+1}$ & - \\
\hline
\end{tabular}

Hence $r^{*}=1$, from which we deduce that

$$
r_{2}(H)=\mu+r^{*}-3=t+2+1-3=t .
$$

2. If $\left(\frac{p_{i}}{\ell}\right)=1$ for all $i \in\{1, \ldots, t\}$, then the prime ideals of $k$ which ramify in $\mathbb{K}$ are $(\sqrt{\ell}), 2, \wp_{i}$, and $\bar{\wp}_{i}$, where $p \mathcal{O}_{k}=\wp_{i} \bar{\wp}_{i}, i=1, \ldots, t$. Proceeding as in the cases above, we get the following table.

\begin{tabular}{|c|c|c|c|c|}
\hline Unit/Character & $\sqrt{\ell}$ & $\wp_{i}$ & $\bar{\wp}_{i}$ & 2 \\
\hline-1 & + & - & - & + \\
\hline$\epsilon$ & - & $\left(\frac{p_{i}}{\ell}\right)_{4}\left(\frac{\ell}{p_{i}}\right)_{4}$ & $-\left(\frac{p_{i}}{\ell}\right)_{4}\left(\frac{\ell}{p_{i}}\right)_{4}$ & $(-1)^{t+1}$ \\
\hline$-\epsilon$ & - & $-\left(\frac{p_{i}}{\ell}\right)_{4}\left(\frac{\ell}{p_{i}}\right)_{4}$ & $\left(\frac{p_{i}}{\ell}\right)_{4}\left(\frac{\ell}{p_{i}}\right)_{4}$ & $(-1)^{t+1}$ \\
\hline
\end{tabular}

Hence $r^{*}=0$, from which we deduce that

$$
r_{2}(H)=\mu+r^{*}-3=2 t+2+0-3=2 t-1 .
$$

Assume $n=\delta \prod_{i=1}^{t_{1}} p_{i} \prod_{j=1}^{t_{2}} q_{j}$, with $t_{1}+t_{2}$ is even, or $n=2 \prod_{i=1}^{t_{1}} p_{i} \prod_{j=1}^{t_{2}} q_{j}$, with $t_{1}+t_{2}$ is odd, where $p_{i} \equiv q_{j} \equiv 3(\bmod 4)$ and $\left(\frac{p_{i}}{\ell}\right)=-\left(\frac{q_{j}}{\ell}\right)=-1$, for all $i \in$ $\left\{1, \ldots, t_{1}\right\}$ and $j \in\left\{1, \ldots, t_{2}\right\}$, then according to the previous discussion we obtain $r^{*}=0$, and thus $r_{2}(H)=t_{1}+2 t_{2}-1$.

3.5. Fifth case: $n=\prod_{i=1}^{i=t} p_{i} \prod_{j=1}^{j=s} q_{j}, p_{i} \equiv-q_{j} \equiv 1(\bmod 4) \forall(i, j)$ and $s$ is odd.

Theorem 3.5. Let $\mathbb{K}=\mathbb{Q}\left(\sqrt{n \epsilon_{0} \sqrt{\ell}}\right)$ be a real cyclic quartic number field, where $\ell \equiv 5(\bmod 8)$ is a positive prime integer, $n$ a square-free positive integer relatively prime to $\ell$ and $\epsilon_{0}$ the fundamental unit of the quadratic subfield $k=\mathbb{Q}(\sqrt{\ell})$. Assume $n=\prod_{i=1}^{i=t} p_{i} \prod_{j=1}^{j=s} q_{j}$ with $p_{i} \equiv-q_{j} \equiv 1(\bmod 4)$ for all $(i, j) \in\{1, \ldots, t\} \times\{1, \ldots, s\}$ and $s$ is odd. Denote by $h$ the number of prime ideals of $k$ dividing all the $p_{i}^{\prime} s$, $i \in\{1, \ldots, t\}$.

1. If, for all $j,\left(\frac{q_{j}}{\ell}\right)=-1$, then $r_{2}(H)=h+s-1$.

2. If, for all $j,\left(\frac{q_{j}}{\ell}\right)=1$, then $r_{2}(H)=h+2 s-2$.

Moreover, if $\prod_{j=1}^{j=s} q_{j}=\prod_{i=1}^{i=s_{1}} q_{i} \prod_{j=s_{1}+1}^{j=s} q_{j}$ with $s$ odd, $q_{i} \equiv q_{j} \equiv 3(\bmod 4)$ and $\left(\frac{q_{i}}{\ell}\right)=$ $-\left(\frac{q_{j}}{\ell}\right)=-1$, for all $i \in\left\{1, \ldots, s_{1}\right\}$ and $j \in\left\{s_{1}+1, \ldots, s\right\}$, then $r_{2}(H)=h+s_{1}+$ $2\left(s-s_{1}\right)-2$.

Proof. Proceeding as above, we have four cases to discus.

1. If $\left(\frac{p_{i}}{\ell}\right)=\left(\frac{q_{j}}{\ell}\right)=-1$, for all $i=1, \ldots, t$ and for all $j=1, \ldots, s$, then the prime ideals of $k$ which ramify in $\mathbb{K}$ are $(\sqrt{\ell}), \mathfrak{p}_{i}$ and $\mathfrak{q}_{j}$, the prime ideals of $k$ above $p_{i}$ and $q_{j}$ respectively, i.e. $\mu=t+s+1$. Proceeding as above, we get the following table. 


\begin{tabular}{|c|c|c|c|}
\hline Unit/Character & $\sqrt{\ell}$ & $\mathfrak{p}_{i}$ & $\mathfrak{q}_{j}$ \\
\hline-1 & + & + & + \\
\hline$\epsilon$ & - & + & - \\
\hline$-\epsilon$ & - & + & - \\
\hline
\end{tabular}

Thus $r^{*}=1$, from which we deduce that

$$
r_{2}(H)=\mu+r^{*}-3=t+s+1+1-3=t+s-1 .
$$

2. If $\left(\frac{p_{i}}{\ell}\right)=-\left(\frac{q_{j}}{\ell}\right)=1$, for all $i=1, \ldots, t$ and for all $j=1, \ldots, s$, then the prime ideals of $k$ which ramify in $\mathbb{K}$ are $(\sqrt{\ell}), \wp_{i}, \bar{\wp}_{i}$ and $\mathfrak{q}_{j}$, where $p \mathcal{O}_{k}=\wp_{i} \bar{\wp}_{i}$ and $\mathfrak{q}_{j}$ is the prime ideal of $k$ above $q_{j}$, i.e. $\mu=2 t+s+1$. Proceeding as above, we get the following results:

\begin{tabular}{|c|c|c|c|c|}
\hline Unit/Character & $\sqrt{\ell}$ & $\wp_{i}$ & $\bar{\wp}_{i}$ & $\mathfrak{q}_{j}$ \\
\hline-1 & + & + & + & + \\
\hline$\epsilon$ & - & $\left(\frac{p_{i}}{\ell}\right)_{4}\left(\frac{\ell}{p_{i}}\right)_{4}$ & $\left(\frac{p_{i}}{\ell}\right)_{4}\left(\frac{\ell}{p_{i}}\right)_{4}$ & - \\
\hline$-\epsilon$ & - & $\left(\frac{p_{i}}{\ell}\right)_{4}\left(\frac{\ell}{p_{i}}\right)_{4}$ & $\left(\frac{p_{i}}{\ell}\right)_{4}\left(\frac{\ell}{p_{i}}\right)_{4}$ & - \\
\hline
\end{tabular}

Thus $r^{*}=1$, this result implies that

$$
r_{2}(H)=\mu+r^{*}-3=2 t+s+1+1-3=2 t+s-1 .
$$

3. If $\left(\frac{p_{i}}{\ell}\right)=-\left(\frac{q_{j}}{\ell}\right)=-1$, for all $i=1, \ldots, t$ and for all $j=1, \ldots, s$, then the prime ideals of $k$ which ramify in $\mathbb{K}$ are $(\sqrt{\ell}), \mathfrak{p}_{i}, \rho_{j}$, and $\bar{\rho}_{j}$, where $q_{j} \mathcal{O}_{k}=\rho_{j} \bar{\rho}_{j}$ and $\mathfrak{p}_{j}$ is the prime ideal of $k$ above $p_{j}$, i.e. $\mu=t+2 s+1$. Proceeding as above, we get the following table:

\begin{tabular}{|c|c|c|c|c|}
\hline Unit/Character & $\sqrt{\ell}$ & $\mathfrak{p}_{i}$ & $\rho_{j}$ & $\bar{\rho}_{j}$ \\
\hline-1 & + & + & - & - \\
\hline$\epsilon$ & - & + & $\left(\frac{q_{j}}{\ell}\right)_{4}\left(\frac{\ell}{q_{j}}\right)_{4}$ & $-\left(\frac{q_{j}}{\ell}\right)_{4}\left(\frac{\ell}{q_{j}}\right)_{4}$ \\
\hline$-\epsilon$ & - & + & $-\left(\frac{q_{j}}{\ell}\right)_{4}\left(\frac{\ell}{q_{j}}\right)_{4}$ & $\left(\frac{q_{j}}{\ell}\right)_{4}\left(\frac{\ell}{q_{j}}\right)_{4}$ \\
\hline
\end{tabular}

Thus $r^{*}=0$, this implies that

$$
r_{2}(H)=\mu+r^{*}-3=t+2 s+1+0-3=t+2 s-2 .
$$

4. If $\left(\frac{p_{i}}{\ell}\right)=\left(\frac{q_{j}}{\ell}\right)=1$, for all $i=1, \ldots, t$ and for all $j=1, \ldots, s$, then the prime ideals of $k$ which ramify in $\mathbb{K}$ are $(\sqrt{\ell}), \wp_{i}, \bar{\wp}_{i}, \rho_{j}$ and $\bar{\rho}_{j}$, where $p_{i} \mathcal{O}_{k}=\wp_{i} \bar{\wp}_{i}$ and $q_{j} \mathcal{O}_{k}=\rho_{j} \bar{\rho}_{j}$, i.e. $\mu=2 t+2 s+1$. Proceeding as above, we get the following table:

\begin{tabular}{|c|c|c|c|c|c|}
\hline Unit/Character & $\sqrt{\ell}$ & $\wp_{i}$ & $\bar{\wp}_{i}$ & $\rho_{j}$ & $\bar{\rho}_{j}$ \\
\hline-1 & + & + & + & - & - \\
\hline$\epsilon$ & - & $\left(\frac{p_{i}}{\ell}\right)_{4}\left(\frac{\ell}{p_{i}}\right)_{4}$ & $-\left(\frac{p_{i}}{\ell}\right)_{4}\left(\frac{\ell}{p_{i}}\right)_{4}$ & $\left(\frac{q_{j}}{\ell}\right)_{4}\left(\frac{\ell}{q_{j}}\right)_{4}$ & $\left(\frac{q_{j}}{\ell}\right)_{4}\left(\frac{\ell}{q_{j}}\right)_{4}$ \\
\hline$-\epsilon$ & - & $-\left(\frac{p_{i}}{\ell}\right)_{4}\left(\frac{\ell}{p_{i}}\right)_{4}$ & $\left(\frac{p_{i}}{\ell}\right)_{4}\left(\frac{\ell}{p_{i}}\right)_{4}$ & $\left(\frac{q_{j}}{\ell}\right)_{4}\left(\frac{\ell}{q_{j}}\right)_{4}$ & $\left(\frac{q_{j}}{\ell}\right)_{4}\left(\frac{\ell}{q_{j}}\right)_{4}$ \\
\hline
\end{tabular}

From which we deduce that $r^{*}=0$, and thus

$$
r_{2}(H)=\mu+r^{*}-3=2 t+2 s+1+0-3=2 t+2 s-2 .
$$

In general, if $\prod_{j=1}^{j=s} q_{j}=\prod_{i=1}^{i=s_{1}} q_{i} \prod_{j=s_{1}+1}^{j=s} q_{j}$, with $s$ is an odd integer, $q_{i} \equiv q_{j} \equiv 3(\bmod 4)$ and $\left(\frac{q_{i}}{\ell}\right)=-\left(\frac{q_{j}}{\ell}\right)=-1, i \in\left\{1, \ldots, s_{1}\right\}, j \in\left\{1, \ldots, s_{2}\right\}$, then taking into account 
the discussions above one gets $r^{*}=0$, and thus

$$
r_{2}(H)=h+s_{1}+2 s_{2}+1+0-3=h+s_{1}+2\left(s-s_{1}\right)-2,
$$

where $h$ is always the number of the prime divisors of all the $p_{i}{ }^{\prime} \mathrm{s}, p_{i} \equiv 1(\bmod 4), i \in$ $\{1, \ldots, t\}$ in $k$.

3.6. Sixth case: $n=\delta \prod_{i=1}^{i=t} p_{i} \prod_{j=1}^{j=s} q_{j}, s$ is even, or $n=2 \prod_{i=1}^{i=t} p_{i} \prod_{j=1}^{j=s} q_{j}, s$ is odd, where $p_{i} \equiv-q_{j} \equiv 1(\bmod 4)$ for all $(i, j)$.

Theorem 3.6. Let $\mathbb{K}=\mathbb{Q}\left(\sqrt{n \epsilon_{0} \sqrt{\ell}}\right)$ be a real cyclic quartic number field, where $\ell \equiv 5(\bmod 8)$ is a positive prime integer, $n$ a square-free positive integer relatively prime to $\ell$ and $\epsilon_{0}$ the fundamental unit of the quadratic subfield $k=\mathbb{Q}(\sqrt{\ell})$. Assume $n=\delta \prod_{i=1}^{i=t} p_{i} \prod_{j=1}^{j=s} q_{j}$ with $s$ even or $n=2 \prod_{i=1}^{i=t} p_{i} \prod_{j=1}^{j=s} q_{j}$ with s odd, where $p_{i} \equiv-q_{j} \equiv 1$ $(\bmod 4)$, for all $(i, j) \in\{1, \ldots, t\} \times\{1, \ldots, s\}$, are prime integers. Denote by $h$ the number of the prime ideals of $k$ above all the $p_{i}^{\prime} s, i \in\{1, \ldots, t\}$.

1. If, for all $j,\left(\frac{q_{j}}{\ell}\right)=-1$, then $r_{2}(H)=h+s$.

2. If, for all $j,\left(\frac{q_{j}}{\ell}\right)=1$, then $r_{2}(H)=h+2 s-1$.

Moreover, if $\prod_{j=1}^{j=s} q_{j}=\prod_{i=1}^{i=s_{1}} q_{i} \prod_{j=s_{1}+1}^{j=s} q_{j}$ with $\left(\frac{q_{i}}{\ell}\right)=-\left(\frac{q_{j}}{\ell}\right)=-1$, for all $i \in\left\{1, \ldots, s_{1}\right\}$ and $j \in\left\{s_{1}+1, \ldots, s\right\}$, assuming $s$ even if $n=\delta \prod_{i=1}^{i=t} p_{i} \prod_{j=1}^{j=s} q_{j}$ and odd if $n=$ $2 \prod_{i=1}^{i=t} p_{i} \prod_{j=1}^{j=s} q_{j}$, then $r_{2}(H)=h+s_{1}+2\left(s-s_{1}\right)-1$.

Proof. There are also four cases to distinguish:

1. If $\left(\frac{p_{i}}{\ell}\right)=\left(\frac{q_{j}}{\ell}\right)=-1$, for all $i \in\{1, \ldots, t\}$ and $j \in\{1, \ldots, s\}$, then the prime ideals of $k$ which ramify in $\mathbb{K}$ are $2,(\sqrt{\ell}), \mathfrak{p}_{i}$ and $\mathfrak{q}_{j}$. Proceeding as in the first cases above, we get the following table:

\begin{tabular}{|c|c|c|c|c|}
\hline Unit/Character & $\sqrt{\ell}$ & 2 & $\mathfrak{p}_{i}$ & $\mathfrak{q}_{j}$ \\
\hline-1 & + & + & + & + \\
\hline$\epsilon$ & - & $(-1)^{s-1}$ & + & - \\
\hline$-\epsilon$ & - & $(-1)^{s-1}$ & + & - \\
\hline
\end{tabular}

Hence $r^{*}=1$, and

$$
r_{2}(H)=\mu+r^{*}-3=t+s+2+1-3=t+s .
$$

2. If $\left(\frac{p_{i}}{\ell}\right)=-\left(\frac{q_{j}}{\ell}\right)=1$, for all $i \in\{1, \ldots, t\}$ and $j \in\{1, \ldots, s\}$, then the prime ideals of $k$ which ramify in $\mathbb{K}$ are $2,(\sqrt{\ell}), \wp_{i}, \bar{\wp}_{i}$ and $\mathfrak{q}_{j}$, where $p_{i} \mathcal{O}_{k}=\wp_{i} \bar{\wp}_{i}$. Proceeding as in the first cases above, we get the following table:

\begin{tabular}{|c|c|c|c|c|c|}
\hline Unit/Character & $\sqrt{\ell}$ & 2 & $\wp_{i}$ & $\bar{\wp}_{i}$ & $\mathfrak{q}_{j}$ \\
\hline-1 & + & + & + & + & + \\
\hline$\epsilon$ & - & $(-1)^{s-1}$ & $\left(\frac{p_{i}}{\ell}\right)_{4}\left(\frac{\ell}{p_{i}}\right)_{4}$ & $\left(\frac{p_{i}}{\ell}\right)_{4}\left(\frac{\ell}{p_{i}}\right)_{4}$ & - \\
\hline$-\epsilon$ & - & $(-1)^{s-1}$ & $\left(\frac{p_{i}}{\ell}\right)_{4}\left(\frac{\ell}{p_{i}}\right)_{4}$ & $\left(\frac{p_{i}}{\ell}\right)_{4}\left(\frac{\ell}{p_{i}}\right)_{4}$ & - \\
\hline
\end{tabular}


Hence $r^{*}=1$, and

$$
r_{2}(H)=\mu+r^{*}-3=2 t+s+2+1-3=2 t+s .
$$

3. If $\left(\frac{p_{i}}{\ell}\right)=-\left(\frac{q_{j}}{\ell}\right)=-1$; for all $i \in\{1, \ldots, t\}$ and $j \in\{1, \ldots, s\}$, then the prime ideals of $k$ which ramify in $\mathbb{K}$ are $2,(\sqrt{\ell}), \mathfrak{p}_{i}, \rho_{j}$, and $\bar{\rho}_{j}$, where $q_{j} \mathcal{O}_{k}=\rho_{j} \bar{\rho}_{j}$. Proceeding as in the first cases above, we get the following table:

\begin{tabular}{|c|c|c|c|c|c|}
\hline Unit/Character & $\sqrt{\ell}$ & 2 & $\mathfrak{p}_{i}$ & $\rho_{j}$ & $\bar{\rho}_{j}$ \\
\hline-1 & + & + & + & - & - \\
\hline$\epsilon$ & - & $(-1)^{s-1}$ & + & $\left(\frac{q_{j}}{\ell}\right)_{4}\left(\frac{\ell}{q_{j}}\right)_{4}$ & $-\left(\frac{q_{j}}{\ell}\right)_{4}\left(\frac{\ell}{q_{j}}\right)_{4}$ \\
\hline$-\epsilon$ & - & $(-1)^{s-1}$ & + & $-\left(\frac{q_{j}}{\ell}\right)_{4}\left(\frac{\ell}{q_{j}}\right)_{4}$ & $\left(\frac{q_{j}}{\ell}\right)_{4}\left(\frac{\ell}{q_{j}}\right)_{4}$ \\
\hline
\end{tabular}

Hence $r^{*}=0$, and

$$
r_{2}(H)=\mu+r^{*}-3=t+2 s+2+0-3=t+2 s-1 .
$$

4. If $\left(\frac{p_{i}}{\ell}\right)=\left(\frac{q_{j}}{\ell}\right)=1$, for all $i \in\{1, \ldots, t\}$ and $j \in\{1, \ldots, s\}$, then the prime ideals of $k$ which ramify in $\mathbb{K}$ are $2,(\sqrt{\ell}), \wp_{i}, \bar{\wp}_{i}, \rho_{j}$ and $\bar{\rho}_{j}$, where $p_{i} \mathcal{O}_{k}=\wp_{i} \bar{\wp}_{i}$, $q_{j} \mathrm{O}_{k}=\rho_{j} \bar{\rho}_{j}$. Proceeding as in the first cases above, we get the following table:

\begin{tabular}{|c|c|c|c|c|c|c|}
\hline Unit/Chara & $\sqrt{\ell}$ & 2 & $\wp_{i}$ & $\bar{\wp}_{i}$ & $\rho_{j}$ & $\bar{\rho}_{j}$ \\
\hline-1 & + & + & + & + & - & - \\
\hline$\epsilon$ & - & $(-1)^{s-1}$ & $\left(\frac{p_{i}}{\ell}\right)_{4}\left(\frac{\ell}{p_{i}}\right)_{4}$ & $\left(\frac{p_{i}}{\ell}\right)_{4}\left(\frac{\ell}{p_{i}}\right)_{4}$ & $\left(\frac{q_{j}}{\ell}\right)_{4}\left(\frac{\ell}{q_{j}}\right)_{4}$ & $-\left(\frac{q_{j}}{\ell}\right)_{4}\left(\frac{\ell}{q_{j}}\right)_{4}$ \\
\hline$-\epsilon$ & - & $(-1)^{s-1}$ & $\left(\frac{p_{i}}{\ell}\right)_{4}\left(\frac{\ell}{p_{i}}\right)_{4}$ & $\left(\frac{p_{i}}{\ell}\right)_{4}\left(\frac{\ell}{p_{i}}\right)_{4}$ & $-\left(\frac{q_{j}}{\ell}\right)_{4}\left(\frac{\ell}{q_{j}}\right)_{4}$ & $\left(\frac{q_{j}}{\ell}\right)_{4}\left(\frac{\ell}{q_{j}}\right)_{4}$ \\
\hline
\end{tabular}

Hence $r^{*}=0$, and

$$
r_{2}(H)=\mu+r^{*}-3=2 t+2 s+2+0-3=2 t+2 s-1 .
$$

In general, if $\prod_{j=1}^{j=s} q_{j}=\prod_{i=1}^{i=s_{1}} q_{i} \prod_{j=s_{1}+1}^{j=s} q_{j}$ with $\left(\frac{q_{i}}{\ell}\right)=-\left(\frac{q_{j}}{\ell}\right)=-1$, for all $i \in\left\{1, \ldots, s_{1}\right\}$ and $j \in\left\{s_{1}+1, \ldots, s\right\}$, assuming $s$ even if $n=\delta \prod_{i=1}^{i=t} p_{i} \prod_{j=1}^{j=s} q_{j}$ and odd if $n=$ $2 \prod_{i=1}^{i=t} p_{i} \prod_{j=1}^{j=s} q_{j}$, then taking into account the discussions above one gets $r^{*}=0$, and thus $r_{2}(H)=h+s_{1}+2\left(s-s_{1}\right)+2+0-3=h+s_{1}+2\left(s-s_{1}\right)-1$.

\section{Applications}

In this section, we shall determine the integers $n$ satisfying $r_{2}(H)$, the rank of the 2-class group $H$ of $\mathbb{K}=\mathbb{Q}\left(\sqrt{n \epsilon_{0} \sqrt{\ell}}\right)$, is equal to $0,1,2$ or 3 . For this we adopt the following notations: $p$ and $p_{i}$ (resp. $q$ and $q_{i}$ ), $i \in \mathbb{N}^{*}$, are prime integers congruent to 1 (resp. 3) modulo $4 . \delta=1$ or 2 . The following theorems are simple deductions from the results of the previous sections.

Theorem 4.1. Let $\mathbb{K}=\mathbb{Q}\left(\sqrt{n \epsilon_{0} \sqrt{\ell}}\right)$ be a real cyclic quartic number field, where $\ell \equiv 5(\bmod 8)$ is a positive prime integer, $n$ a square-free positive integer relatively prime to $\ell$ and $\epsilon_{0}$ the fundamental unit of the quadratic subfield $k=\mathbb{Q}(\sqrt{\ell})$. The class number of $\mathbb{K}$ is odd if and only if one of the following assertions holds: 
1. $n=1$ or 2 .

2. $n$ is a prime integer congruent to $3(\bmod 4)$.

Numerical Examples 4.1. For all the examples below, we use PARI/GP calculator version 2.9.1 (64bit), Nov 22, 2016.

1. For $n=1$ and $\ell=173 \equiv 5(\bmod 8), H$ is trivial, the class number of the class group of $\mathbb{K}=\mathbb{Q}\left(\sqrt{\epsilon_{0} \sqrt{\ell}}\right)$ is 5 .

For $n=2$ and $\ell=197 \equiv 5(\bmod 8), H$ is trivial, in reality the class group of $\mathbb{K}=\mathbb{Q}\left(\sqrt{2 \epsilon_{0} \sqrt{\ell}}\right)$ is of type $(3,3)$.

2. For $n=q=67 \equiv 3(\bmod 4)$ and $\ell=53 \equiv 5(\bmod 8)$, we have $\left(\frac{q}{\ell}\right)=-1$ and $H$ is trivial, the class number of the class group of $\mathbb{K}=\mathbb{Q}\left(\sqrt{67 \epsilon_{0} \sqrt{\ell}}\right)$ is 17 .

For $n=q=79 \equiv 3(\bmod 4)$ and $\ell=13 \equiv 5(\bmod 8)$, we have $\left(\frac{q}{\ell}\right)=1$ and the class group of $\mathbb{K}=\mathbb{Q}\left(\sqrt{79 \epsilon_{0} \sqrt{\ell}}\right)$ is trivial.

Theorem 4.2. Let $\mathbb{K}=\mathbb{Q}\left(\sqrt{n \epsilon_{0} \sqrt{\ell}}\right)$ be a real cyclic quartic number field, where $\ell \equiv 5(\bmod 8)$ is a prime, $n$ a square-free positive integer relatively prime to $\ell$ and $\epsilon_{0}$ the fundamental unit of the quadratic subfield $k=\mathbb{Q}(\sqrt{\ell})$. H is cyclic if and only if one of the following assertions holds:

1. $n=\delta p$ with $\left(\frac{p}{\ell}\right)=-1$.

2. $n=2 q$.

3. $n=p q$ with $\left(\frac{p}{\ell}\right)=-1$.

Numerical Examples 4.2. Here are some examples.

1. For $n=p=13 \equiv 1(\bmod 4)$ and $\ell=37 \equiv 5(\bmod 8)$, we have $\left(\frac{p}{\ell}\right)=-1$ and $H$ is cyclic of order 2 .

For $n=p=17 \equiv 1(\bmod 4)$ and $\ell=29 \equiv 5(\bmod 8)$, we have $\left(\frac{p}{\ell}\right)=-1$ and $H$ is cyclic of order 2 .

2. For $n=2 p=2.41 \equiv 2(\bmod 4)$ and $\ell=13 \equiv 5(\bmod 8)$, we have $\left(\frac{p}{\ell}\right)=-1$ and $H$ is cyclic of order 2 .

For $n=2 p=2.51 \equiv 2(\bmod 4)$ and $\ell=61 \equiv 5(\bmod 8)$, we have $\left(\frac{p}{\ell}\right)=-1$ and $H$ is cyclic of order 2 .

3. For $n=2 q=2.19 \equiv-2(\bmod 4)$ and $\ell=53 \equiv 5(\bmod 8)$, we have $H$ is cyclic of order 2 .

4. For $n=p q=5.7 \equiv 3(\bmod 4)$ and $\ell=53 \equiv 5(\bmod 8)$, we have $\left(\frac{p}{\ell}\right)=-1$, $\left(\frac{p}{q}\right)=-1$ and $H$ is cyclic of order 2 .

For $n=p q=5.11 \equiv 3(\bmod 4)$ and $\ell=13 \equiv 5(\bmod 8)$, we have $\left(\frac{p}{\ell}\right)=-1$, $\left(\frac{p}{q}\right)=1$ and $H$ is cyclic of order 2 .

Theorem 4.3. Let $\mathbb{K}=\mathbb{Q}\left(\sqrt{n \epsilon_{0} \sqrt{\ell}}\right)$ be a real cyclic quartic number field, where $\ell \equiv 5(\bmod 8)$ is a prime, $n$ a square-free positive integer relatively prime to $\ell$ and $\epsilon_{0}$ the fundamental unit of the quadratic subfield $k=\mathbb{Q}(\sqrt{\ell})$. The rank $r_{2}(H)$ equals 2 if and only if $n$ takes one of the following forms.

1. $n=\delta p$ and $\left(\frac{p}{\ell}\right)=1$.

2. $n=\delta p_{1} p_{2}$ and $\left(\frac{p_{1}}{\ell}\right)=\left(\frac{p_{2}}{\ell}\right)=-1$.

3. $n=p q$ and $\left(\frac{p}{\ell}\right)=1$.

4. $n=2 p q$ and $\left(\frac{p}{\ell}\right)=-1$.

5. $n=p_{1} p_{2} q$ and $\left(\frac{p_{1}}{\ell}\right)=\left(\frac{p_{2}}{\ell}\right)=-1$.

6. $n=\delta q_{1} q_{2}$ and at least one of the two symbols $\left(\frac{q_{1}}{\ell}\right),\left(\frac{q_{2}}{\ell}\right)$ equals -1 . 
7. $n=q_{1} q_{2} q_{3}$ and at most one of the symbols $\left(\frac{q_{1}}{\ell}\right),\left(\frac{q_{2}}{\ell}\right),\left(\frac{q_{3}}{\ell}\right)$ equals 1 .

Numerical Examples 4.3. Here are some examples.

1. For $n=p=13 \equiv 1(\bmod 4)$ and $\ell=101 \equiv 5(\bmod 8)$, we have $\left(\frac{p}{\ell}\right)=1$ and $H$ is of type $(2,2)$.

For $n=2 p=2.73 \equiv 2(\bmod 4)$ and $\ell=109 \equiv 5(\bmod 8)$, we have $\left(\frac{p}{\ell}\right)=1$ and $H$ is of type $(2,4)$.

2. For $n=p_{1} p_{2}=17.37 \equiv 1(\bmod 4)$ and $\ell=29 \equiv 5(\bmod 8)$, we have $\left(\frac{p_{1}}{\ell}\right)=-1$, $\left(\frac{p_{2}}{\ell}\right)=-1$ and $H$ is of type $(2,2)$.

3. For $n=p q=13.11 \equiv 3(\bmod 4)$ and $\ell=53 \equiv 5(\bmod 8)$, we have $\left(\frac{p}{\ell}\right)=1$ and $H$ is of type $(2,2)$.

4. For $n=2 p q=2.17 .11 \equiv-2(\bmod 4)$ and $\ell=29 \equiv 5(\bmod 8)$, we have $\left(\frac{p}{\ell}\right)=-1$ and $H$ is of type $(2,2)$.

5. For $n=p_{1} p_{2} q=17.37 .23 \equiv 3(\bmod 4)$ and $\ell=61 \equiv 5(\bmod 8)$, we have $\left(\frac{p_{1}}{\ell}\right)=-1,\left(\frac{p_{2}}{\ell}\right)=-1$ and $H$ is of type $(2,2)$.

6. For $n=q_{1} q_{2}=79.83 \equiv 1(\bmod 4)$ and $\ell=37 \equiv 5(\bmod 8)$, we have $\left(\frac{q_{1}}{\ell}\right)=-1$, $\left(\frac{q_{2}}{\ell}\right)=1$ and $H$ is of type $(2,2)$. For $n=2 q_{1} q_{2}=2.47 .59 \equiv 2(\bmod 4)$ and $\ell=13 \equiv 5(\bmod 8)$, we have $\left(\frac{q_{1}}{\ell}\right)=-1,\left(\frac{q_{2}}{\ell}\right)=-1$ and $H$ is of type $(2,2)$.

7. For $n=q_{1} q_{2} q_{3}=23.71 .83 \equiv 3(\bmod 4)$ and $\ell=61 \equiv 5(\bmod 8)$, we have $\left(\frac{q_{1}}{\ell}\right)=-1,\left(\frac{q_{2}}{\ell}\right)=-1,\left(\frac{q_{3}}{\ell}\right)=1$ and $H$ is of type $(2,2)$.

Theorem 4.4. Let $\mathbb{K}=\mathbb{Q}\left(\sqrt{n \epsilon_{0} \sqrt{\ell}}\right)$ be a real cyclic quartic number field, where $\ell \equiv 5(\bmod 8)$ is a prime, $n$ a square-free positive integer relatively prime to $\ell$ and $\epsilon_{0}$ the fundamental unit of the quadratic subfield $k=\mathbb{Q}(\sqrt{\ell})$. The rank $r_{2}(H)$ equals 3 if and only if $n$ takes one of the following forms.

1. $n=\delta p_{1} p_{2}$ and $\left(\frac{p_{1}}{\ell}\right)=-\left(\frac{p_{2}}{\ell}\right)=1$.

2. $n=\delta p_{1} p_{2} p_{3}$ and $\left(\frac{p_{i}}{\ell}\right)=-1$ for all $i \in\{1,2,3\}$.

3. $n=2 p q$ and $\left(\frac{p}{\ell}\right)=1$.

4. $n=\delta q_{1} q_{2}$ and $\left(\frac{q_{i}}{\ell}\right)=1$ for all $i \in\{1,2\}$.

5. $n=q_{1} q_{2} q_{3}$ and only one of the symbols $\left(\frac{q_{i}}{\ell}\right), i \in\{1,2,3\}$, is -1 .

6. $n=2 q_{1} q_{2} q_{3}$ and at most one of the symbols $\left(\frac{q_{i}}{\ell}\right), i \in\{1,2,3\}$, is 1 .

7. $n=p_{1} p_{2} q$ and $\left(\frac{p_{1}}{\ell}\right)=-\left(\frac{p_{2}}{\ell}\right)=1$.

8. $n=2 p_{1} p_{2} q$ and $\left(\frac{p_{1}}{\ell}\right)=\left(\frac{p_{2}}{\ell}\right)=-1$.

9. $n=\delta p q_{1} q_{2}$ and $\left(\frac{p}{\ell}\right)=-1$ and at least one of the symbols $\left(\frac{q_{i}}{\ell}\right), i \in\{1,2\}$, is -1 .

10. $n=p q_{1} q_{2} q_{3}$ and $\left(\frac{p}{\ell}\right)=-1$ and at most one of the symbols $\left(\frac{q_{i}}{\ell}\right), i \in\{1,2,3\}$, is 1 .

11. $n=p_{1} p_{2} p_{3} q$ and $\left(\frac{p_{i}}{\ell}\right)=-1$ for all $i \in\{1,2,3\}$.

Numerical Examples 4.4. Here are some examples.

1. For $n=p_{1} p_{2}=37.89 \equiv 1(\bmod 4)$ and $\ell=5 \equiv 5(\bmod 8)$, we have $\left(\frac{p_{1}}{\ell}\right)=-1$, $\left(\frac{p_{2}}{\ell}\right)=1$ and $H$ is of type $(2,4,4)$.

For $n=2 p_{1} p_{2}=2.41 .53 .89 \equiv 2(\bmod 4)$ and $\ell=29 \equiv 5(\bmod 8)$, we have $\left(\frac{p_{1}}{\ell}\right)=-1,\left(\frac{p_{2}}{\ell}\right)=1$ and $H$ is of type $(2,2,4)$.

2. For $n=p_{1} p_{2} p_{3}=17.53 .89 \equiv 1(\bmod 4)$ and $\ell=61 \equiv 5(\bmod 8)$, we have $\left(\frac{p_{1}}{\ell}\right)=-1,\left(\frac{p_{2}}{\ell}\right)=-1,\left(\frac{p_{3}}{\ell}\right)=-1$ and $H$ is of type $(2,2,2)$.

For $n=2 p_{1} p_{2} p_{3}=2.17 .61 .89 \equiv 2(\bmod 4)$ and $\ell=29 \equiv 5(\bmod 8)$, we have $\left(\frac{p_{1}}{\ell}\right)=-1,\left(\frac{p_{2}}{\ell}\right)=-1,\left(\frac{p_{3}}{\ell}\right)=-1$ and $H$ is of type $(2,2,2)$.

3. For $n=2 p q=2.53 .79 \equiv-2(\bmod 4)$ and $\ell=37 \equiv 5(\bmod 8)$, we have $\left(\frac{p}{\ell}\right)=1$ and $H$ is of type $(2,2,4)$. 
4. For $n=2 q_{1} q_{2}=2.59 .83 \equiv 2(\bmod 4)$ and $\ell=29 \equiv 5(\bmod 8)$, we have $\left(\frac{q_{1}}{\ell}\right)=$ $\left(\frac{q_{2}}{\ell}\right)=-1$ and $H$ is of type $(2,2,4)$.

For $n=q_{1} q_{2}=67.71 \equiv 1(\bmod 4)$ and $\ell=37 \equiv 5(\bmod 8)$, we have $\left(\frac{q_{1}}{\ell}\right)=$ $\left(\frac{q_{2}}{\ell}\right)=-1$ and $H$ is of type $(2,2,2)$.

5. For $n=q_{1} q_{2} q_{3}=19.47 .71 \equiv-1(\bmod 4)$ and $\ell=37 \equiv 5(\bmod 8)$, we have $\left(\frac{q_{1}}{\ell}\right)=-1,\left(\frac{q_{2}}{\ell}\right)=\left(\frac{q_{3}}{\ell}\right)=1$ and $H$ is of type $(2,2,2)$.

6. For $n=2 q_{1} q_{2} q_{3}=2.7 .67 .71 \equiv-2(\bmod 4)$ and $\ell=53 \equiv 5(\bmod 8)$, we have $\left(\frac{q_{1}}{\ell}\right)=1,\left(\frac{q_{2}}{\ell}\right)=\left(\frac{q_{3}}{\ell}\right)=-1$ and $H$ is of type $(2,2,2)$.

7. For $n=p_{1} p_{2} q=13.17 .43 \equiv 3(\bmod 4)$ and $\ell=61 \equiv 5(\bmod 8)$, we have $\left(\frac{p_{1}}{\ell}\right)=-1,\left(\frac{p_{2}}{\ell}\right)=1$ and $H$ is of type $(2,4,4)$.

8. For $n=2 p_{1} p_{2} q=2.29 .53 .79 \equiv 2(\bmod 4)$ and $\ell=61 \equiv 5(\bmod 8)$, we have $\left(\frac{p_{1}}{\ell}\right)=\left(\frac{p_{2}}{\ell}\right)=-1$ and $H$ is of type $(2,2,2)$.

9. For $n=p q_{1} q_{2}=37.47 .71 \equiv 1(\bmod 4)$ and $\ell=5 \equiv 5(\bmod 8)$, we have $\left(\frac{p}{\ell}\right)=-1$, $\left(\frac{q_{1}}{\ell}\right)=-1,\left(\frac{q_{2}}{\ell}\right)=1$ and $H$ is of type $(2,2,2)$.

For $n=2 p q_{1} q_{2}=2.17 .31 .83 \equiv 2(\bmod 4)$ and $\ell=37 \equiv 5(\bmod 8)$, we have $\left(\frac{p}{\ell}\right)=-1,\left(\frac{q_{1}}{\ell}\right)=-1,\left(\frac{q_{2}}{\ell}\right)=1$ and $H$ is of type $(2,2,2)$.

10. For $n=p q_{1} q_{2} q_{3}=5.43 .31 .71 \equiv 3(\bmod 4)$ and $\ell=13 \equiv 5(\bmod 8)$, we have $\left(\frac{p}{\ell}\right)=-1,\left(\frac{q_{1}}{\ell}\right)=1,\left(\frac{q_{2}}{\ell}\right)=\left(\frac{q_{3}}{\ell}\right)=-1$ and $H$ is of type $(2,2,2)$.

11. For $n=p_{1} p_{2} p_{3} q=13.17 .29 .83 \equiv 3(\bmod 4)$ and $\ell=37 \equiv 5(\bmod 8)$, we have $\left(\frac{p_{1}}{\ell}\right)=\left(\frac{p_{2}}{\ell}\right)=\left(\frac{p_{3}}{\ell}\right)=-1$ and $H$ is of type $(2,2,2)$.

Remark 4.1. If the integer $n$ does not take any value in Theorems 4.1, 4.2, 4.3, 4.4, then $r_{2}(H) \geq 4$.

\section{ACKNOWLEDGMENT}

We would like to thank the unknown referee for his/her several helpful suggestions and for calling our attention to the missing details.

\section{REFERENCES}

[1] A. Aziz. Sur le 2-groupe de classes de certains corps de nombres, Ann. Sci. Maht. Québec 28, no. 1-2, (2004), 37-44.

[2] A. Azizi and A. Mouhib, Sur le rang du 2-groupe de classes de $\mathbb{Q}(\sqrt{m}, \sqrt{d})$ où $m=2$ ou un premier $p \equiv 1 \bmod 4$, Trans. Amer. Math. Soc. 353, No 7 (2001), 2741-2752.

[3] A. Azizi and A. Mouhib, Le 2-rang du groupe de classes de certains corps biquadratiques et applications, Int. J. Math. vol 15, No. 02, (2004), 169-182.

[4] A. Baker, An introduction to Galois theory, Lecture note (2013).

[5] H. Bauer, Zur Berechnung der 2-Klassenzahl der quadratischen Zahlkörper mit genau zwei verschieden Diskriminantenprimteilern, J. reine angew. Math. 248 (1971), 42-46.

[6] E. Brown and C. J. Parry, The 2-class group of certain biquadratic number fields, J. reine angew. Math., vol 295, (1977) 61-71.

[7] E. Brown and C. J. Parry, The 2-class group of certain biquadratic number fields II, Pacific J. Math. vol 78, No. 1, (1978), 61-71.

[8] M. N. Gras, Table numérique du nombre de classes et des unités des extensions cycliques réelles de degré 4 de $\mathbb{Q}$, Publ. Math. Fac. Sciences Bensançon, Théorie des Nombres (197778).

[9] M. N. Gras, Calcul du nombre de classes et des unités des extensions abéliennes réelles de $\mathbb{Q}$, Bull. Sci. Math. 101 (1977), 97-129.

[10] M. N. Gras, Classes et unités des extensions cycliques réelles de degré 4 de $\mathbb{Q}$, Ann. Inst. Fourier (Grenoble) 29 (1979), 107-124.

[11] H. Hasse, Arithmetishe Bestimmung von Grundeinheit und Klassenzahl in zyklischen kubischen und biquadratishen Zahlkorpern, Abh. Deutsche Akad. Wiss. Berlin, Math, no 2, (1948) 1-95. 
[12] H. Hasse, An algorithm for determining the structure of the 2-Sylow-subgroup of the divisor class group of a quadratic number fleld, Symposia. Mat. 15 (1975), 341-352.

[13] K. Hardy, R. H. Hudson, D. Richman, K. S. Williams, and M. Holtz, Calculation of the class numbers of imaginary cyclic quartic fileds, Journal. Math. comp. vol 49, (1987), 615-620.

[14] D. Hilbert, Bericht über den algebraischen Zahlkörper, Jber. Deutsche Math. Verein. 4, 189495.

[15] J. A. Hymo, C. J. Parry, On relative integral bases for cyclic quartic fields, J. Number Theory, vol 34, Issue 2, (1990), 189-197.

[16] H. W. Leopoldt, Uber einheitengruppe und klassenzahl reeller abelscher zahlkörper, Abh. Deutsche Akad. Wiss. Berlin. Math. 2, (1953), 1-48.

[17] T. M. McCall, C. J. Parry and R. R. Ranalli, Imaginary bicyclic biquadratic fields with cyclic 2-class group, J. Number. Theor, 53 (1995), 88-99.

[18] T. M. McCall, C. J. Parry and R. R. Ranalli, The 2-rank of the class group of imaginary bicyclic biquadratic fields, Can. J. Math. vol 49, (1997), 283-300.

[19] C. J. Parry, Pure quartic number fields whose class numbers are even, J. reine angew. Math. vol 264, (1975), 102-112.

[20] C. J. Parry, Real quadratic fields with class number divisible by 5, Math. Comput. vol 31, (1977), 1019-1029.

[21] C. J. Parry, A genus theory for quartic fields, J. reine. angew. Math. 314, (1980), 40-71.

[22] Zh. Xianke, Cyclic quartic fields and genus theory of their subfields, J. Number Theory, no 18, (1984), 350-355.

[23] O. Zink, Extension cycliques de degré $2^{n}$ sur $\mathbb{Q}$, Séminaire delange-Pisot-Poitou. Théorie des nombres, Tome 8, no 2, exp. no 16, (1966-1967), 1-12.

Abdelmalek Azizi: Mohammed First University, Sciences Faculty, Mathematics Department, Oujda, Morocco

E-mail address: abdelmalekazizi@yahoo.fr

Mohammed Tamimi: Mohammed First University, Sciences Faculty, Mathematics Department, Oujda, Morocco

E-mail address: med.tamimi@gmail.com

Abdelkader Zekhnini: Mohammed First University, Pluridisciplinary Faculty, Mathematics Department, Nador, Morocco

E-mail address: zekha1@yahoo.fr 